\title{
Magnetic charge and moment dynamics in artificial kagome spin ice
}

\author{
A. Farhan, ${ }^{1,2,3,{ }^{*}}$ P. M. Derlet, ${ }^{4, \dagger}$ L. Anghinolff, ${ }^{1,2}$ A. Kleibert, ${ }^{5}$ and L. J. Heyderman ${ }^{1,2, \ddagger}$ \\ ${ }^{1}$ Paul Scherrer Institute, Switzerland, 5232 Villigen PSI, Switzerland \\ ${ }^{2}$ Laboratory for Mesoscopic Systems, Department of Materials, ETH Zurich, 8093 Zurich, Switzerland \\ ${ }^{3}$ Advanced Light Source, Lawrence Berkeley National Laboratory, 1 Cyclotron Road, Berkeley, California 94720, USA \\ ${ }^{4}$ Condensed Matter Theory Group, NUM, Paul Scherrer Institute, 5232 Villigen, Switzerland \\ ${ }^{5}$ Swiss Light Source, Paul Scherrer Institute, 5232 Villigen, Switzerland
}

(Received 4 December 2015; revised manuscript received 7 July 2017; published 7 August 2017)

\begin{abstract}
Spin ice materials represent an intriguing class of frustrated magnetic systems which, through their geometry, admit an exponential number of approximately degenerate configurations. In this paper, the relaxation properties of a thermally active artificial kagome spin ice system are studied. Through application of an external magnetic field, an out-of-equilibrium vertex charge ordered configuration is selected and relaxed under approximate zero-field conditions. Using x-ray photo-emission electron microscopy, the magnetic moment and vertex charge degrees of freedom are followed in space and time, revealing different dynamics to that seen in past athermal equilibration protocols, and a relaxation which is well described by a point-dipolar model system. Furthermore the charge correlations are found to relax with a time scale several times smaller than that of the moment correlations.
\end{abstract}

DOI: 10.1103/PhysRevB.96.064409

\section{INTRODUCTION}

Frustration in matter is a phenomenon that arises when the interactions within a given system cannot be simultaneously minimized resulting in a very large number of low-energy configurations with an approximate degeneracy that scales exponentially with system size. Prominent examples are the way protons arrange themselves in water ice [1] and how adjacent spins, because of the magnetic interactions and crystal structure, orient themselves in a magnetic material according to an ice rule [2-4]. The pyrochlore spin ice [2-4] and the two-dimensional kagome sublattices of herbertsmithite variants [5] are well-known systems, which exhibit such spin ice physics. In these materials, a perfect degeneracy would lead to a finite configurational entropy as the temperature approaches zero-Pauling's residual entropy [1] — and thus a zero-point disordered ground state. However, when more distant interactions are present, this perfect degeneracy can be lifted, introducing new energy scales and therefore temperature regimes at which less disordered phases become thermodynamically stable, reducing or even extinguishing Pauling's residual entropy - a phenomenon that has been demonstrated for the $\mathrm{Dy}_{2} \mathrm{Ti}_{2} \mathrm{O}_{7}$ pyrochlore system [6].

Kagome spin ice is a two-dimensional lattice of spins positioned on the sites of the kagome lattice, in which the local ice rule constraint [7] forces each nearest-neighbor triplet of spins to configurations in which two point away/towards and one points towards/away from the triplet's center of position vertex-see Fig. 1. When viewed in terms of the net magnetic flux entering or leaving each triplet vertex, the kagome system may be represented by a hexagonal array of magnetic vertex charges, $Q= \pm q$, that reside at the vertices where three nanomagnets meet [see Fig. 1(a)]. This

\footnotetext{
*alan.farhan@gmx.net

†peter.derlet@psi.ch

${ }^{\ddagger}$ laura.heyderman@psi.ch
}

system can be partially realized at the microscopic level in pyrochlore spin ice in the presence of a magnetic field [8], and at the mesoscopic level via a two-dimensional patterned array of nanomagnets, the individual shape anisotropies of which lead to single domain magnets with Ising-like moments [9-17]. These latter systems offer the advantage that their magnetization dynamics can be studied in real space and time on the level of individual magnetic moments to complement reciprocal space techniques, which have been used to probe the pyrochlore spin ice systems in the past. Theory has shown that, if the spins of the kagome system interact via the long-range magnetic dipolar interaction, the degeneracy of the spin ice sector is lifted giving rise to three low-temperature phases $[18,19]$. In order of decreasing temperature, the different regimes can be listed as a magnetic moment and vertex charge disordered phase (spin ice I), a vertex charge ordered and partially ordered magnetic moment phase (spin ice II) in which there is moment fragmentation into both a magnetic charge crystal and a Coulomb phase [20,21], and a lowest-temperature magnetic moment and vertex charge long-range ordered (LRO) phase [see Fig. 1(b)].

In earlier work, low-energy magnetic configurations in artificial spin ice systems could only be obtained athermally through demagnetization protocols $[9,11]$ or with thermal treatments prior to measurement [15]. Here, various microscopy methods were implemented to perform real-space imaging of the magnetic configurations and, in addition to attempts to obtain low-energy states, the creation and separation of emergent magnetic monopole/antimonopole pairs could be observed during magnetization reversal [10]. More recently, it was shown to be possible to manufacture thermally active artificial kagome spin ice from thinner films and observe in real space and time the exploration of the low-energy magnetic configurations in finite structures $[12,13]$. By tuning the nanomagnet volume, the blocking temperature of the individual nanomagnets can be engineered to be well below the critical temperatures associated with the phase transitions of extended systems. In such an artificial kagome ice system, 
(a)

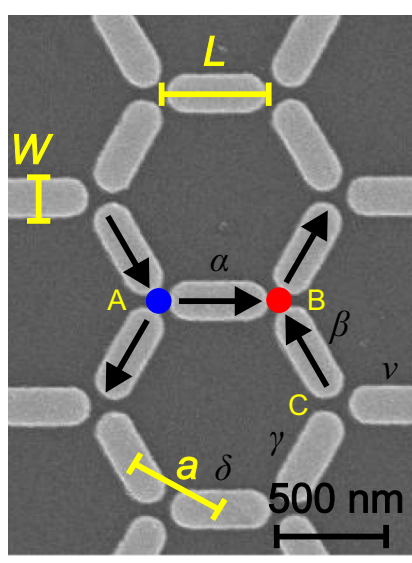

(b)

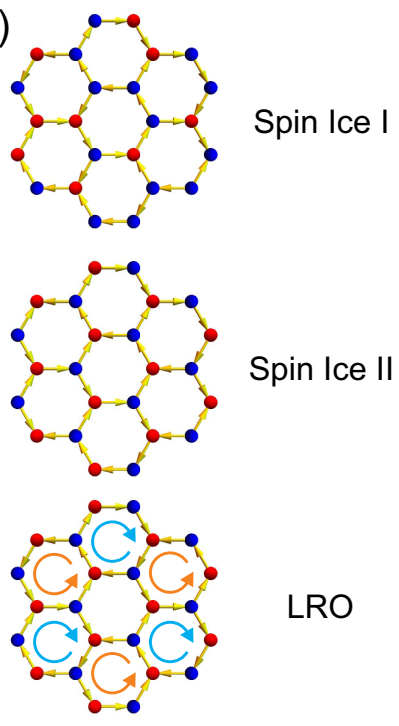

FIG. 1. (a) Scanning electron microscope image of part of an artificial kagome spin ice consisting of nanomagnets with a length $L$ $=470 \mathrm{~nm}$, width $W=170 \mathrm{~nm}$, and thickness $d=3 \mathrm{~nm}$ arranged on a lattice with nearest-neighbor center-to-center distance $a=$ $500 \mathrm{~nm}$. Due to their elongated shape and the small value of $d$, each nanomagnet is well described by an Ising moment, which points parallel or antiparallel to its long axis. Superimposed on the image are two nearest-neighbor triplets of Ising moments that adhere to the ice rule of two in(out) moments and one out(in) moments. The net local flux may be identified as magnetic vertex charges of either $Q=q$ or $-q$ (red or blue circles) existing on the parent honeycomb lattice. (b) Upper schematic: At high enough temperatures these vertex charges, like the underlying Ising moments, are spatially disordereda phase referred to as spin ice I. Middle schematic: As the temperature is lowered, the vertex charges order and the spin ice II phase is formed. Lower schematic: At even lower temperatures, the long-range nature of the underlying dipolar interaction gives rise to ordered moments, resulting in a long-range ordered phase where a partial tiling of microvortices defines the zero-temperature ground state.

dynamical signatures of both phase transitions could be obtained indirectly via low-energy muon spectroscopy methods [22].

In the present paper, a thermally active artificial kagome spin ice system is realized and studied at a temperature in which the spin ice I phase is expected to be at equilibrium. Using X-ray photo-emission electron microscopy, the associated magnetic degrees of freedom of the artificial kagome spin ice are resolved both spatially and temporarily allowing us to study the early stages of relaxation to the spin ice I phase from a prepared out-of-equilibrium spin ice II charge ordered configuration. Kinetic Monte Carlo (KMC) simulations are used to compare the experimentally observed relaxation dynamics with the predictions of a simplified point-dipolar model system. The sample preparation and experimental measurement methods, the use of moment and charge-correlation measures, and the nature of the numerical simulations are detailed in Sec. II. In Sec. III, the experimental results including the time dependence of the correlation functions and comparison with simulation are presented. Specifically, we observe a dynamical creation and destruction of charge and moment order which

is fundamentally different from the behavior observed in past athermal work $[10,23]$ and we experimentally confirm that the dynamics of such artificial spin systems are controlled by the dipolar interaction between the patterned nanomagnets.

\section{METHODS}

\section{A. Sample fabrication and characterization}

We used electron-beam lithography to pattern a Permalloy $\left(\mathrm{Ni}_{80} \mathrm{Fe}_{20}\right)$ wedge film on a silicon (100) substrate. Following exposure and development of a 70-nm-thick polymethylmethacrylate (PMMA) resist spin coated on a silicon (100) substrate, a Permalloy film wedge, along with a 3-nm capping layer of aluminum to protect against oxidation, was deposited by thermal evaporation at room temperature at a rate of several nanometers per minute and a base pressure of $3 \times 10^{-6} \mathrm{mbar}$. The unwanted resist and magnetic material were then removed in acetone by ultrasound assisted liftoff. Extended arrays $(50 \times 40 \mu \mathrm{m})$ of artificial kagome spin ice consisting of nanomagnets with length $L=470 \mathrm{~nm}$, width $W=170 \mathrm{~nm}$, and lattice parameter $a=500 \mathrm{~nm}$ [see Fig. 1(a)] were fabricated with thicknesses $d$ increasing from zero up to $15 \mathrm{~nm}$ over a distance of $4 \mathrm{~mm}$. Similar fabrication procedures have been used to construct thermally active artificial square ice systems [24,25]. The magnetic imaging is performed at a film thickness of $3 \mathrm{~nm}$, where we observe thermally driven moment reorientations at a time scale of several minutes at $T=420 \mathrm{~K}$. Each nanomagnet is small enough to be single domain and its elongated shape forces the moments to point into one of two possible directions along the nanomagnet long axis, thus mimicking a single Ising macrospin.

Magnetic imaging was performed using a photo-emission electron microscope (PEEM), employing x-ray magnetic circular dichroism (XMCD) at the Fe $L_{3}$ edge [26-28]. The dark and bright contrast in the magnetic contrast maps (referred to as XMCD images) is a measure of the orientation of the magnetic moment of a nanomagnet relative to the $\mathrm{x}$-ray polarization vector. Nanomagnets with magnetic moments pointing towards the $\mathrm{x}$-ray propagation vector will appear dark, while nanomagnets with magnetic moments opposing the $\mathrm{x}$ ray propagation vector will appear bright [see Figs. 2(a)-2(c)].

\section{B. Magnetic moment and vertex charge correlation functions}

A quantitative measure of the magnetic moment and vertex charge dynamics in the experimental or simulation data can be gained through the determination of two-point magnetic moment and vertex charge correlation functions, defined, respectively, as

$$
\mathrm{CM}_{i j}(t, \tau)=\left\langle\hat{\mathbf{m}}_{i}(t) \cdot \hat{\mathbf{m}}_{i+j}(t+\tau)\right\rangle
$$

and

$$
\mathrm{CQ}_{i j}(t, \tau)=\left\langle Q_{i}(t) Q_{i+j}(t+\tau)\right\rangle .
$$

Here, the indices $i$ and $j$ label the magnetic moment directions $\hat{\mathbf{m}}$ or vertex charges $Q, i+j$ refers to the $j$ th neighbor of $i$, and $\langle\cdot\rangle$ represents a spatial average over the experimental or simulation data window.

In Eqs. (1) and (2), $t$ and $t+\tau$ give the two times between which the correlations are observed. Under equilibrium con- 


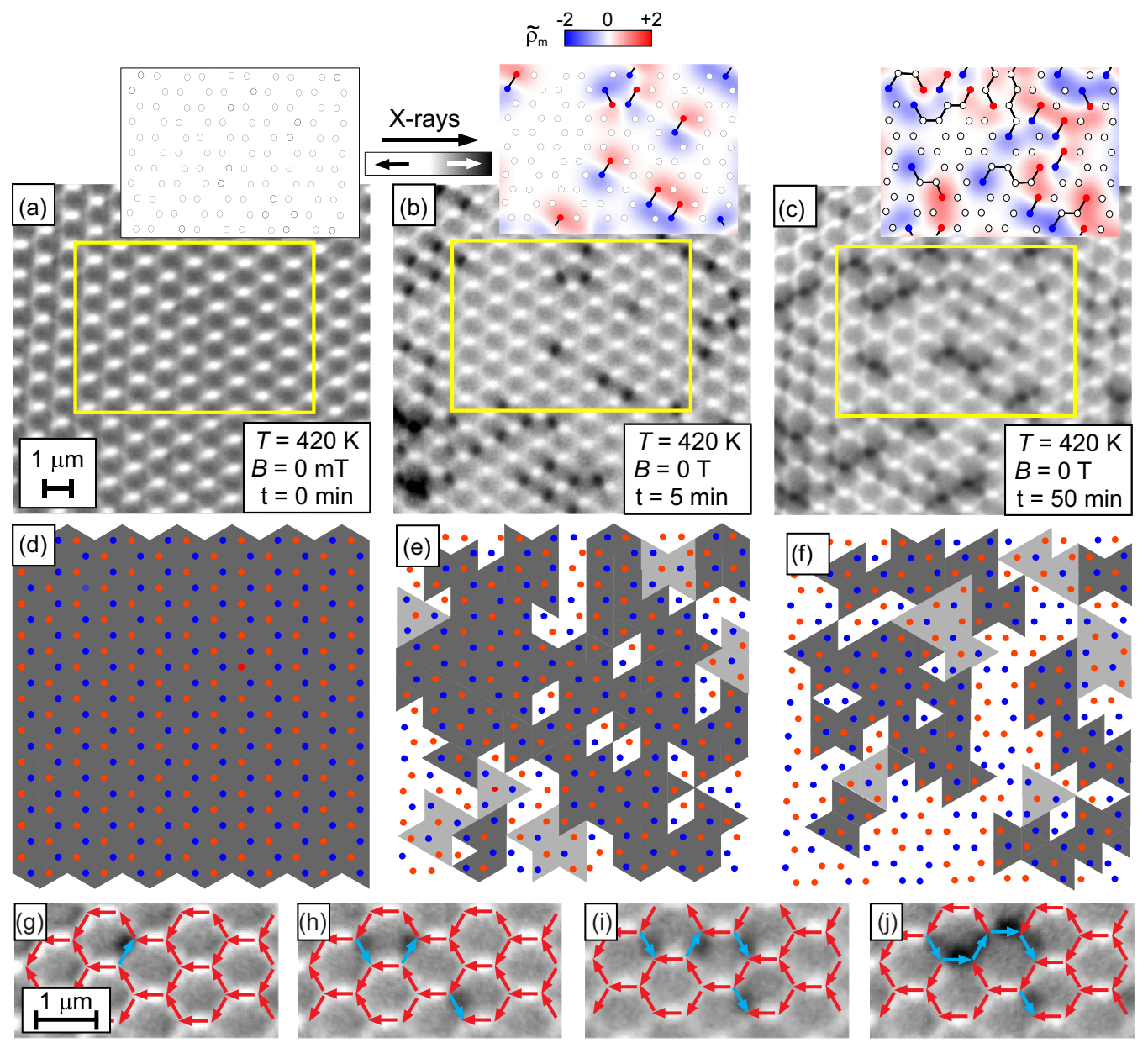

FIG. 2. (a-c) XMCD snapshots of artificial kagome spin ice starting from an initial out-of-equilibrium spin ice II phase, which then relaxes towards the equilibrium spin ice I phase. The insets show the corresponding smeared charge-density maps indicating the creation of emergent magnetic monopoles (see caption of Fig. 3), which remain confined due to a strict obedience to the local ice rule at each vertex. In these insets, the red and blue points reflect charge defects that can be seen as emergent magnetic monopoles connected by Dirac strings consisting of chains of magnetic moments connecting the charge defect pair (black lines). (d-f) The corresponding $Q=+q /-q$ red and blue vertex charge configurations, which show how the initially global charge order of the prepared state decays into regions of local charge order characteristic of spin ice I. The two shades of gray refer to the two possible local (central vertex plus nearest neighbors) charge order configurations. $(\mathrm{g}-\mathrm{j}) \mathrm{XMCD}$ image sequence highlighting a typical example of thermally induced Dirac-string evolution in artificial kagome spin ice under a strict obedience to the local ice rule.

ditions the correlation functions do not depend on $t$. However, during relaxation there will be a dependence on $t$, which is the time since relaxation began. Two temporal correlation relations will be presently considered. In the first case, the correlations as a function of $t$ with $\tau=0$ minutes will be measured. These instantaneous correlation functions allow for a quantitative comparison to the equilibrium (spin ice I) instantaneous values. In the second case, the correlations as a function of $\tau$ with $t=0$ will be measured, which gives quantitative information on how the system decorrelates from the initial charge ordered configuration.

For the case of the instantaneous ( $\tau=0)$ magnetic moment correlation function, a coarse-grained version is used in which $\operatorname{sgn}\left[\hat{\mathbf{m}}_{i}(t) \cdot \hat{\mathbf{m}}_{i+j}(t)\right]$ is now averaged $[9,11,12,15]$ :

$$
\mathrm{CM}_{i j}^{\mathrm{cg}}(0)=\left\langle\operatorname{sgn}\left[\hat{\mathbf{m}}_{i}(t) \cdot \hat{\mathbf{m}}_{i+j}(t)\right]\right\rangle .
$$

In the above moment correlation functions, we refer to the spatial indices as $\alpha \alpha$ for onsite, $\alpha \beta$ for the first-neighbor, $\alpha \gamma$ for the second-neighbor, and $\alpha \delta$ and $\alpha \nu$ for the fourthneighbor correlations. For the charge correlation functions, we refer to the spatial indices as $\mathrm{AA}$ for onsite, $\mathrm{AB}$ for the first-neighbor, and $\mathrm{AC}$ for the second-neighbor correlations. This nomenclature is displayed in Fig. 1(a).

\section{Visualization of emergent magnetic monopoles}

The relaxation from an initial out-of-equilibrium spin ice II configuration prepared by application of magnetic field (Sec. III) can be viewed from the perspective of emergent magnetic monopoles. These are visualized by inspecting the deviations away from the charge ordered configuration giving a charge difference map, $\Delta Q= \pm 2 q[10,23]$, or may be visualized by calculating the dimensionless smeared charge density $\tilde{\rho}_{\mathrm{m}}=(\Delta x)^{2} \rho_{\mathrm{m}} / q$, where the smeared charge density 
$\rho_{\mathrm{m}}$ is a convolution of the vertex charge $\left(Q_{i}\right)$ distribution with a Gaussian of full width at half maximum $\Delta x=2 a_{\mathrm{h}}$ [10]. Here, the spatial position of the maximum/minimum value provides the location of the emergent magnetic monopoles. The so-called Dirac strings connecting pairs of emergent magnetic monopoles of opposite sign correspond to chains of reversed magnetic moments transferring the flux from negative to positive emergent charge. Here they are mainly used to identify confined monopole pairs which mediate the early stages of the relaxation seen in Fig. 2. For these emergent magnetic monopoles, blue indicates negative charge and red indicates a positive charge.

We emphasize here that emergent magnetic monopoles should be distinguished from the vertex charges $(Q)$ shown in Fig. 1. An emergent magnetic monopole is identified from the difference in vertex charge measured relative to a reference vertex charge ordered configuration, and occurs when the vertex charge difference is $\Delta Q=2$ (monopole) and $\Delta Q=-2$ (antimonopole). The ice sector may be exited on separation of an emergent monopole/antimonopole pair as seen in Fig. 4.

\section{Simulations}

The experimentally observed dynamics is compared to simulations based on the KMC method [29]. Starting from a given magnetic configuration, all possible transitions from this initial configuration are determined together with their associated transition rates. The transition rates are assumed to follow an Arrhenius type behavior given by $\Gamma=v_{0} \exp (-\beta E)$, where the prefactor $\nu_{0}$ is the attempt frequency, $\beta=1 / k_{\mathrm{B}} T$, and $E=E_{0}+1 / 2\left(E_{\mathrm{f}}-E_{\mathrm{i}}\right)$ is the sum of the energy barrier to reorient an individual nanomagnet $E_{0}$ and half of the difference between configurational energies after and before a given transition $\left(E_{\mathrm{f}}-E_{\mathrm{i}}\right)$. The energy of each moment configuration is calculated using the leading-order point-dipolar interaction

$$
V\left(\mathbf{r}_{i j}, \mathbf{m}_{i}, \mathbf{m}_{j}\right)=-\frac{\mu_{0}}{4 \pi r_{i j}^{3}}\left[3\left(\mathbf{m}_{i} \cdot \hat{\mathbf{r}}_{i j}\right)\left(\mathbf{m}_{j} \cdot \hat{\mathbf{r}}_{i j}\right)-\mathbf{m}_{i} \cdot \mathbf{m}_{j}\right]
$$

where $\mathbf{r}_{i j}$ is the distance vector separating the $i$ th and $j$ th point-dipole magnetic moments, $\mathbf{m}_{i}$ and $\mathbf{m}_{j}$, giving the final Hamiltonian $\sum_{i<j} V\left(\mathbf{r}_{i j}, \mathbf{m}_{i}, \mathbf{m}_{j}\right)$. The point-dipole moment of each nanomagnet is given by the product $M L W d$ where $M$ is the experimentally determined magnetization. In addition, variations in the barrier energy, $E_{0}$, due to intrinsic disorder are included via a uniform distribution of width $2 \sigma$. Good agreement between experiment and simulation could be found with $v_{0}=10^{12} \mathrm{~s}^{-1}, E_{0}=1.26 \mathrm{eV}, \sigma=0.3 \mathrm{eV}$, and $M=$ $350 \mathrm{~A} / \mathrm{m}$ (see also [12]). Converged simulation data are obtained when the dipolar interaction range is truncated to at least five neighbor shells.

For this parametrization, the crossover from hightemperature paramagnetism to the spin ice regime (spin ice I) occurs at a temperature of approximately $500 \mathrm{~K}$. To obtain estimates of the critical temperatures corresponding to the spin ice I/II $\left(T_{\mathrm{c} 1}\right)$ and spin ice II/LRO $\left(T_{\mathrm{c} 2}\right)$ phase transitions, ensemble Monte Carlo simulations are performed for the point-dipolar kagome ice, calculating the heat capacity as a function of temperature. Inspection of this curve (not shown) gives the critical temperatures as $T_{\mathrm{c} 1} \approx 140 \mathrm{~K}$ and $T_{\mathrm{c} 2} \approx 50 \mathrm{~K}$. To obtain the low-temperature phase transition, a cluster MC algorithm was needed (in addition to the usual single site method) to access the relevant fluctuations of the spin ice I phase. See Ref. [22] for more details.

\section{RESULTS}

\section{A. Magnetic moment and charge dynamics}

Experimentally, a perfectly charge ordered initial out-ofequilibrium configuration of the spin ice II phase can be set by applying a saturating external magnetic field parallel to one of the three equivalent symmetry axes of the kagome lattice. When the the external magnetic field is removed, the charged ordered remnant state relaxes towards the equilibrium spin ice I phase, allowing us to study the thermally driven nonequilibrium dynamics [Figs. 2(a)-2(c)]. We find that the relaxation of the initial out-of-equilibrium spin ice II configuration to the equilibrium spin ice I phase is driven by the destruction of the vertex charge order, which can be viewed in terms of the creation of emergent magnetic monopoles [10,23]. Such emergent magnetic monopoles are defined as charge defects with respect to vertex charge order, which gives rise to nonzero values of the smeared charge density [10] [see inset of Figs. 2(a)-2(c)], and can only be created as an emergent monopole-antimonopole pair, connected by a so-called Dirac string [10,23]. A comparison of XMCD images and the corresponding smeared charge-density maps reveals Dirac strings consisting of chains of reversed magnetic moments transferring magnetic flux from the negative to the positive charge defects [black lines connecting the blue and red dots in the insets of Figs. 2(a)-2(c)].

The temporal evolution of the moment configuration reveals that, initially, isolated emergent monopole-antimonopole pairs are created. These remain confined and immobile due to a strict obedience to the local ice rule [see insets of Figs. 2(a)-2(c), Figs. 2(g)-2(j), and also Fig. 3]. Further relaxation involves the continued creation of such confined emergent monopole pairs until a density is reached that more closely reflects the spin ice I phase. As this regime is approached, emergent magnetic monopoles of opposite sign and from different (but neighboring) sites annihilate, resulting in a single emergent monopole/antimonopole pair connected by an extended Dirac string. An example of this process involving two emergent monopole/antimonopole pairs is shown in Figs. 2(g)-2(j), with numerous examples also occurring in Fig. 3. This thermally active nonequilibrium emergent monopole dynamics is very different from that observed under athermal field driven conditions where, upon emergent monopole-antimonopole creation, relaxation is mainly mediated by sequential Dirac string extension, thus repeatedly violating the ice rule constraint $[10,23]$.

Underlying the emergent monopole dynamics is the disordering of the initially ordered vertex charge configuration. Because the global vertex charge order configuration has a degeneracy of two, there can be two local charge order configurations, which are visualized as either dark or light gray regions in Figs. 2(d)-2(f). The initially perfect charge ordered configuration is arbitrarily chosen to be dark gray. Inspection of Figs. 2(d)-2(f) reveals that, as relaxation proceeds, local light 

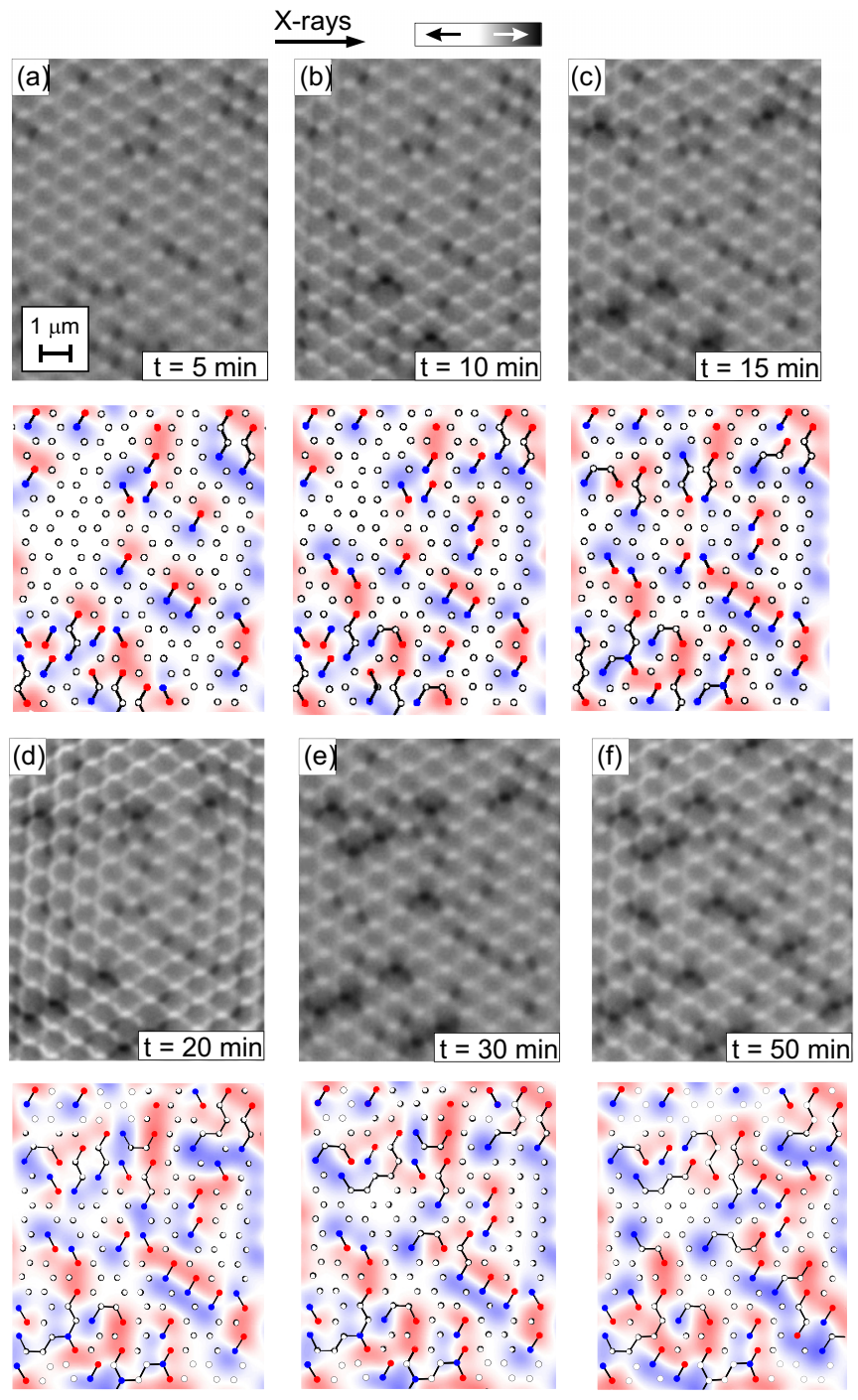

$\Delta Q=0$
$-\Delta Q=-2 q$

$\Delta Q=+2 q$

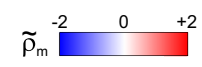

FIG. 3. (a-f) Thermal behavior of emergent magnetic monopoles in artificial kagome spin ice during relaxation to the spin ice I phase. XMCD images (upper panels), obtained as a function of time at constant temperature $T=420 \mathrm{~K}$, are shown together with smeared charge-density maps (lower panels) as well as the emergent monopole/antimonopole pairs $(\Delta Q=+2 q,-2 q, 0$ indicated, respectively, by red, blue, and white dots), where the back lines indicate the connecting Dirac string. It is seen that emergent monopole defects give rise to a nonzero smeared charge density, as in the case of athermal field protocols (see Ref. [10]). However, in contrast to field-driven experiments, each nucleated emergent monopole/antimonopole pair remains confined and immobile due to strict ice rule compliance.

gray regions of "opposite" vertex charge order appear, as well as unshaded regions of local vertex charge disorder. Indeed as the density of emergent monopole-antimonopole pairs increases, the initial perfect vertex charge order breaks up into increasingly smaller domains of alternate (dark and light gray) vertex order until only short-range vertex charge order remains.

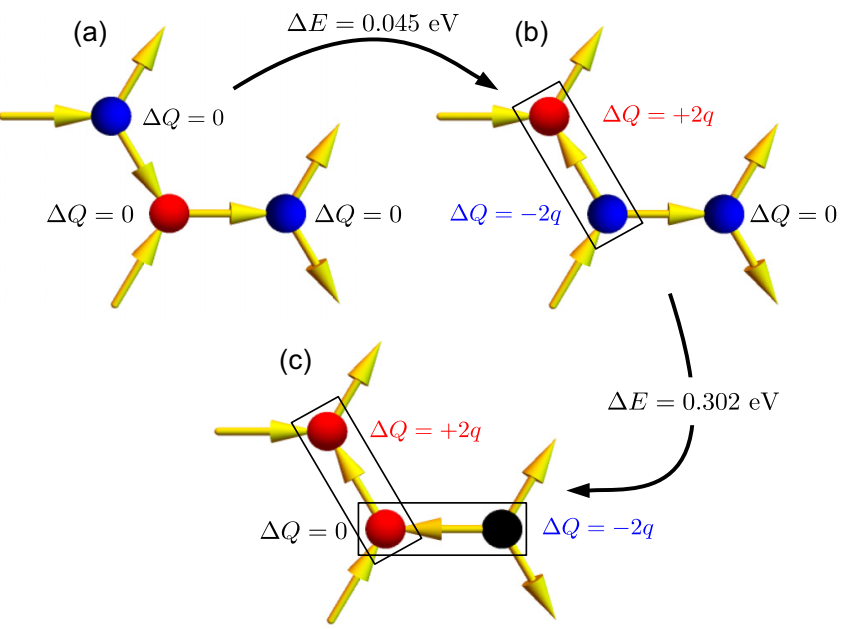

FIG. 4. Three local moment configurations differing by a single moment reversal. The vertex charges are given by the colored balls where blue corresponds to a vertex charge of $-q$, red corresponds to a vertex charge of $+q$, and black corresponds to a vertex charge of $-3 q$. Due to the moment reorientation in going from (a) to (b), there is an exchange of vertex charges and the creation of an emergent monopole/antimonopole pair with charges $\Delta Q= \pm 2 q$. This involves a total change in energy equal to $0.045 \mathrm{eV}$. The small value reflects the fact that the configuration remains within the ice sector. The moment reorientation associated with going from (b) to (c) does, however, violate the ice rule, giving a much larger total change in energy equal to $0.302 \mathrm{eV}$.

The observation of emergent monopole-antimonopole pairs that remain confined can be explained by the point-dipolar model described in Sec. IID. The energetics of the local reorientation of moments, which is required to generate an emergent monopole-antimonopole pair, are shown in Fig. 4. To create an initial emergent monopole-antimonopole pair from the initial out-of-equilibrium spin ice II magnetic state [Fig. 4(a)], one moment is reoriented [Fig. 4(b)]. As a result, there has also been an exchange of the neighboring vertex charge. Via the definitions given in Sec. II C and Ref. [10], this deviation from perfect vertex charge order creates an emergent monopole-antimonopole pair bounded by the rectangular frames in Figs. 4(b) and 4(c).

According to the point-dipolar model described in Sec. II D, the total change in energy due to this reorientation is $0.045 \mathrm{eV}$. In order to propagate one of the emergent magnetic monopoles, a second moment must reorient to achieve the magnetic state shown in Fig. 4(c). However, this leads to a violation of the ice rule (a vertex charge of $-3 q$ is created). The total-energy change associated with this reorientation is $0.302 \mathrm{eV}$. This second reorientation, which is the first step to deconfinement of the pair, is costly in energy and therefore unlikely to occur at the temperature of $420 \mathrm{~K}$ at which the measurements were performed.

The inability to deconfine is also reflected in the corresponding reorientation rates, which control the dynamics in a KMC simulation. The rate of occurrence of a particular moment reorientation is given by $\Gamma=v_{0} \exp \left(-E / k_{\mathrm{B}} T\right)$, where $E=E_{0}+1 / 2\left(E_{\mathrm{f}}-E_{\mathrm{i}}\right)$-see Sec. II D. For the reorientation, which creates the emergent monopole pair at $T=420 \mathrm{~K}$ 


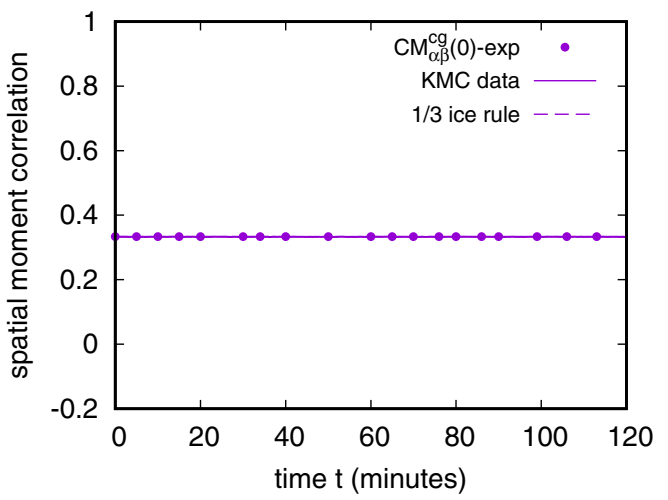

(a)

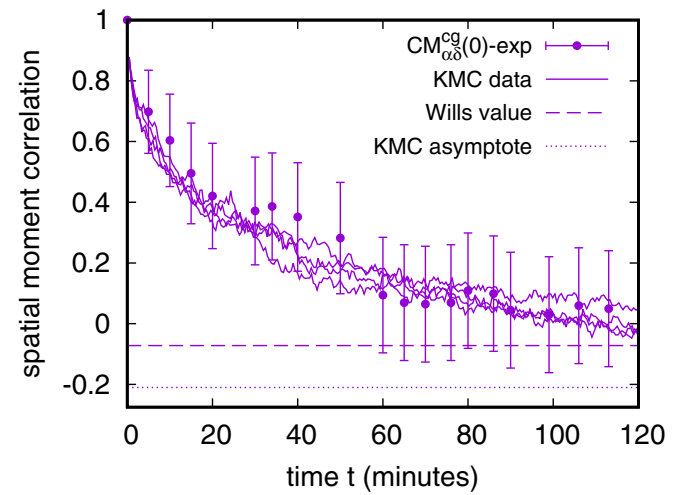

(c)

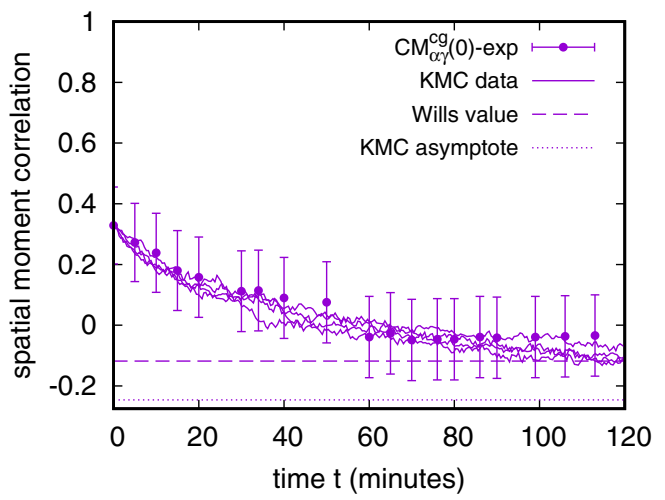

(b)

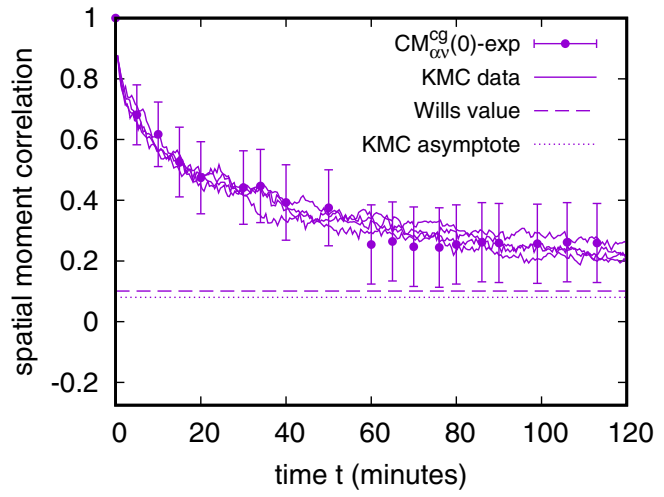

(d)

FIG. 5. Experimentally obtained moment relaxation at $420 \mathrm{~K}$, characterized by instantaneous $(\tau=0)$ course-grained correlation functions [Eq. (3)] for the (a) $\alpha \beta$, (b) $\alpha \gamma$, (c) $\alpha \delta$, and (d) $\alpha v$ neighbor shells [see Fig. 1(a)], plotted as a function of time $t$. At $t=0$ the artificial kagome spin ice is in the out-of-equilibrium moment configuration where all moments are aligned in the direction of the applied saturating field shown in Figs. 2(a) and 2(d) (corresponding to the initial out-of-equilibrium charge ordered state). In all figures, the corresponding connected curves are four KMC simulated relaxation realizations. The dashed lines correspond to the equilibrium values expected for the spin ice I phase derived by Wills et al. [7] and also the KMC asymptotes obtained by extending the KMC simulations to up to $2000 \mathrm{~min}$.

[Figs. 4(a) and 4(b)], the corresponding barrier energy is $E=1.26+0.045 / 2=1.2825 \mathrm{eV}$, giving a rate of $\Gamma=$ $0.024 / \mathrm{min}$ - a value which is characteristic of the reorientation of an individual moment within the spin ice sector. This should be compared with the reorientation rate associated with the ice rule violation [Figs. 4(b) and 4(c)], $\Gamma \simeq 0.0007 / \mathrm{min}$, arising from the larger barrier energy $E=1.26+0.302 / 2=$ $1.411 \mathrm{eV}$. Thus it will be rare for the deconfinement to occur. Moreover, if it were to occur, the barrier energy associated with returning to the nearest-neighbor configuration would be $E=$ $1.26-0.302 / 2=1.109 \mathrm{eV}$, giving a rate of $\Gamma \simeq 3.0 / \mathrm{min}$. In other words, the deconfined pair would return rapidly to its initial confined configuration. Both the high-energy barrier and low reorientation rates explain why deconfinement of the emergent monopole pairs is not seen.

\section{B. Magnetic moment and vertex charge correlation functions}

To quantify the out-of-equilibrium relaxation dynamics, instantaneous ( $\tau=0$ minutes) moment and vertex charge correlation functions are extracted from the XMCD images as a function of time, $t$, after the saturating magnetic field is switched off. Using the coarse-grained moment correlation function up to the third-neighbor shell [see Sec. II B, Eq. (3)], we observe relaxation in the moment spatial correlation func- tions to values that start to approach those characteristic of the spin ice I phase. In Fig. 5, we plot the first-neighbor $\left(\mathrm{CM}_{\alpha \beta}^{\mathrm{cg}}\right)$, second-neighbor $\left(\mathrm{CM}_{\alpha \gamma}^{\mathrm{cg}}\right)$, and third-neighbor $\left(\mathrm{CM}_{\alpha \delta}^{\mathrm{cg}}\right.$ and $\left.\mathrm{CM}_{\alpha \nu}^{\mathrm{cg}}\right)$ coarse-grained moment correlations along with the spin ice I equilibrium values $\left(\mathrm{CM}_{\alpha \gamma}^{\mathrm{cg}}=-0.118, \mathrm{CM}_{\alpha \delta}^{\mathrm{cg}}=-0.072\right.$, and $\mathrm{CM}_{\alpha \nu}^{\mathrm{cg}}=0.101$ ) originally predicted by Wills et al. [7] (see also Ref. [9]) using a nearest-neighbor interaction model. Also shown are the KMC asymptotic values taken directly from the simulations at approximately 1500 minutes, which are somewhat larger and consistent with past work taking into account more distant neighbors [9,11]. Inspection of Fig. 5(a) reveals a nearest-neighbor correlation of precisely $1 / 3$, confirming that the spin ice manifold is never left. The scatter seen in Figs. 5(b)-5(d) is mainly due to the finite field of view of the PEEM/XMCD images. An uncertainty associated with these fluctuations can be estimated by assuming this scatter is statistically independent and normally distributed. Assuming a confidence interval of $95 \%$, the derived uncertainties are included as error bars in Figs. 5(b)-5(d).

For the vertex charge, the corresponding, $\tau=0$, instantaneous nearest- and next-nearest-neighbor correlation functions $\mathrm{CQ}_{\mathrm{AB}}$ and $\mathrm{CQ}_{\mathrm{AC}}$ are shown in Fig. 6. The initial long-range charge order entails a nearest-neighbor charge correlation equal to -1 (each charge has three neighbors of opposite sign) and a next-nearest-neighbor charge correlation equal to 1. 


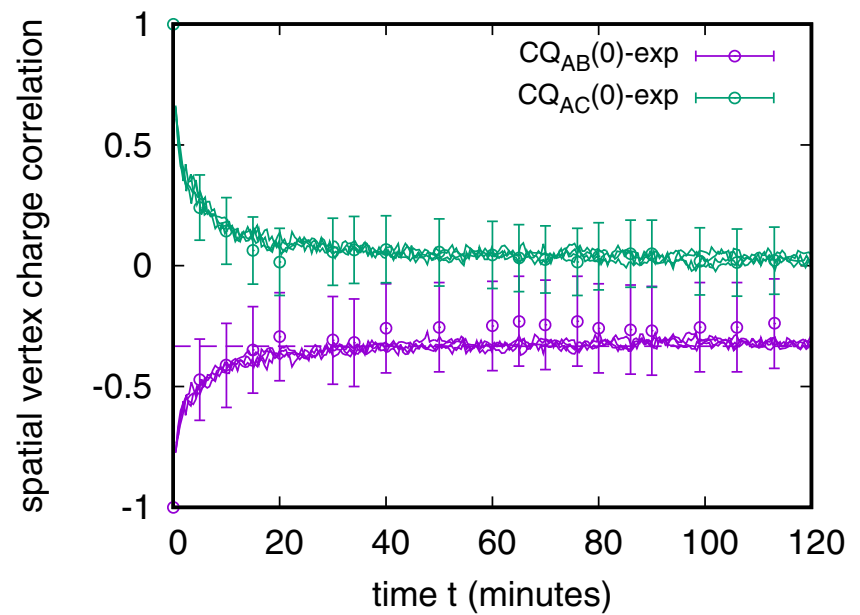

FIG. 6. Experimentally observed vertex charge relaxation characterized by instantaneous $(\tau=0)$ correlation functions [Eq. (2)] plotted as a function of time $(t)$, starting from the initial out-ofequilibrium charge ordered state shown in Figs. 2(a) and 2(d). The connected curves of the same color are four corresponding KMC simulated relaxation realizations. The dashed line associated with the nearest-neighbor vertex charge correlation indicates the expected asymptotic behavior of one-third reflecting local charge neutrality.

During relaxation towards the equilibrium spin ice I phase, the magnitude of the nearest-neighbor charge correlation $\left(\mathrm{CQ}_{\mathrm{AB}}\right)$ decreases and eventually limits to a value of approximately one-third (see purple horizontal dashed line in Fig. 6). This reflects the desire of the system to maintain local charge neutrality at the scale of any charge and its three nearest neighbors. In Fig. 6, the next-nearest-neighbor charge correlation $\left(\mathrm{CQ}_{\mathrm{AC}}\right)$ rapidly becomes negligible due to the lack of long-range charge order of the spin ice I phase. A similar error analysis procedure as that implemented in Fig. 5 is used to derive an error estimate for the experimental vertex charge correlation.

KMC simulations of this relaxation process are also shown in Figs. 5 and 6 and demonstrate good agreement with experiment for both the moment and charge spatial correlation functions. These simulations are performed by beginning with the perfect charge ordered configuration associated with the moments having a component pointing parallel to one of the three equivalent symmetry axes of the kagome lattice (as in the experiment). Four statistically independent KMC simulations of the relaxation are shown using a sample size similar to the experimental field of view. It is seen that all simulation data are well within the confidence limits of the experimental data.

The $\tau=0$ correlation functions shown in Figs. 5 and 6 are a measure of how the instantaneous structure evolves after removal of the external field. To see how the magnetic degrees of freedom decorrelate with the initial out-of-equilibrium spin ice II configuration, the temporal correlation functions of Eqs. (1) and (2) are evaluated as a function of $\tau$, with $t=$ 0 - the time at which the external magnetic field is switched off. This is performed for the moment and charge correlation functions, and for both experiment and simulation. For the moment relaxation [Fig. 7(a)], the initial values depend on the $\tau=0$ moment configuration and the angle between the moments in each neighbor shell [Eq. (1)]. For the charge

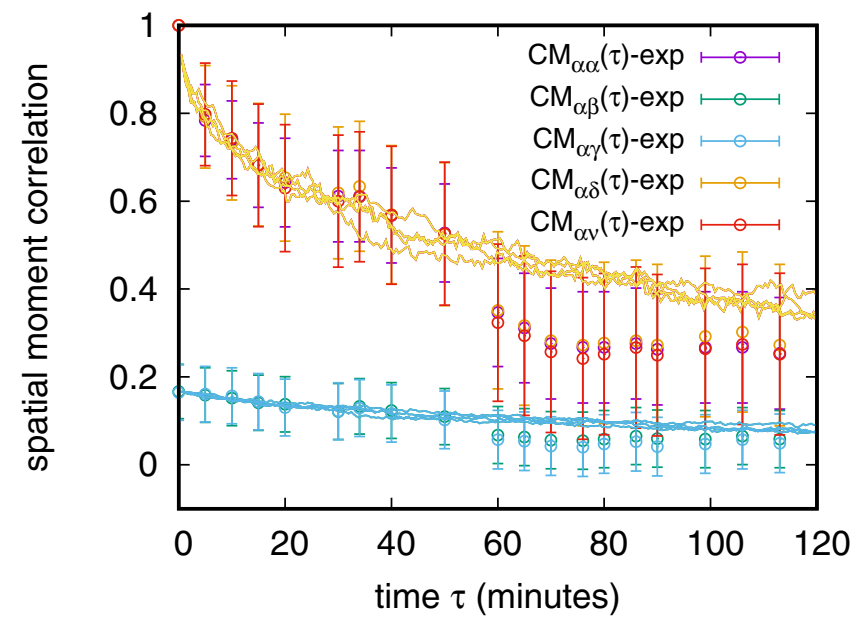

(a)

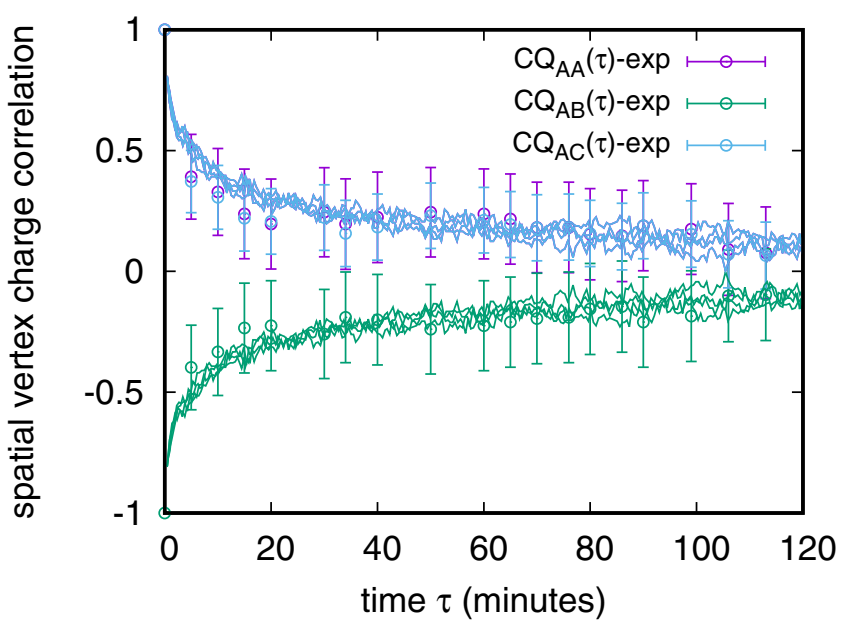

(b)

FIG. 7. Experimentally obtained (a) magnetic moment and (b) vertex charge correlations [Eqs. (1) and (2)] plotted as a function of time, $\tau$, with $t=0$, starting from the initial out-of-equilibrium charge ordered state (at $\tau=0$ ) shown in Figs. 2(a) and 2(d). In both (a) and (b), the connected curves are four corresponding KMC simulated relaxation realizations.

relaxation, the initial sign depends on the initial alternating charge order between neighbor shells. The time scale at which these correlation functions decay to zero indicates the point at which the magnetic structure is statistically decorrelated from the initial configuration and true equilibrium of the spin ice I phase is reached. In Fig. 7(a) we demonstrate that, in terms of the moment degrees of freedom, the last experimental configuration (at approximately $120 \mathrm{~min}$ ) is still far from fully relaxed. Indeed KMC simulations indicate that approximately $1000 \mathrm{~min}$ are needed to obtain a well-equilibrated spin ice I system that is fully decorrelated from the initial out-ofequilibrium spin ice II configuration.

Inspection of Fig. 5 indicates a characteristic moment relaxation time of approximately 30 to $40 \mathrm{~min}$. This compares well to the inverse of a typical moment reorientation rate which does not break the ice rule. For example, the inverse rate associated with reorientation in Figs. 4(a) and 4(b) is $1 / \Gamma=1 / 0.024 \approx 40 \mathrm{~min}$. By comparing the moment and 
charge relaxation time scales in Figs. 5-7, it can be seen that the dynamic time scale of the vertex charges is faster than that of the underlying magnetic moments, which have mostly relaxed within approximately $20 \mathrm{~min}$. This is compatible with the notion that for each charge configuration there is a variety of compatible moment configurations, and therefore more than one reorientation pathway exists between one charge configuration and another. Thus the underlying moment dynamics are expected to result in a faster vertex charge dynamics.

Closer inspection of Fig. 7(a) reveals differences between the experimental and simulated moment correlation functions-beyond $60 \mathrm{~min}$ the experimental data drop abruptly. This deviation is also present in the coarse-grained correlation functions of Figs. 5(b)-5(d), albeit to a lesser extent, where after $60 \mathrm{~min}$ the experimental correlation functions are approximately constant. The observed deviation is unlikely to originate from natural variations due to the finite field of view, since the different KMC simulation realizations in Figs. 5(b)-5(d) differ to an extent that is much less than the deviation in the experiment. One possible source of deviation is the presence of a time-dependent external field at the sample location, which selects a preferred configuration. In addition to the Earth's magnetic field in the PEEM at the Swiss Light Source, which gives an in-plane component of 13-15 $\mu \mathrm{T}$, we have found contributions arising from the sample holder filament used for heating (measured to be $<20 \mu \mathrm{T}$ ) as well as in the surrounding environment (measured to be $<10 \mu \mathrm{T}$ ). Thus, a sporadic magnetic field of up to $40 \mu \mathrm{T}$ can be expected under nominal "zero-field" conditions. Whilst still small, we have seen from our KMC simulations that such fields can modify our artificial kagome spin ice, which has a saturation field of approximately $90 \mathrm{mT}$. This suggests that external stray fields varying at the time scale of several tens of minutes could be responsible for the observed deviation seen in Figs. 5 and 7.

\section{CONCLUDING REMARKS}

We have observed the relaxation of a thermally active mesoscopic kagome ice system from an external-field-selected initial out-of-equilibrium spin ice II configuration towards the equilibrium spin ice I phase in a small but non-negligible external field. The magnetic degrees of freedom are fully resolvable both spatially and temporarily using x-ray PEEM, and the dynamics can be understood within the framework of a model normally associated with microscopic moments. In particular, we have demonstrated the following:

(1) We can satisfactorily rationalize the observed relaxation of an initial out-of-equilibrium spin ice II configuration towards the spin ice I phase with a point-dipolar interaction [Eq. (4)] between the nanomagnets.

(2) The time scale characterizing vertex charge relaxation is shorter by a factor of approximately two when compared to the underlying magnetic moment relaxation (Figs. 5 and 6), reflecting that each charge configuration can arise from a number of different magnetic moment configurations.

(3) Thermal relaxation from a nonequilibrium spin ice I configuration, which strictly adheres to the ice rule leading to emergent magnetic monopole confinement (Fig. 2 and Fig. 3), is fundamentally different from driven relaxation via athermal protocols [10,23].

In summary, a thermally active artificial kagome spin ice system has been realized and studied at a temperature for which spin ice $\mathrm{I}$ is the equilibrium phase. By applying an external magnetic field, an initial charge ordered out-of-equilibrium spin ice II configuration is created and allowed to relax under approximate zero-field conditions. This relaxation has been quantified through the calculation of spatial and temporal magnetic moment and vertex charge correlations, and a pointdipole moment model is found to describe well the observed relaxation. The present paper demonstrates that thermally active artificial kagome spin ice can exhibit complex and collective dynamics, which is well described by a simplified model system normally associated with microscopic degrees of freedom. Such thermally active magnetic metamaterials offer a flexibility not possible in microscopic magnetic systems, and can be tailor made to address both fundamental and applied aspects of magnetism.

\section{ACKNOWLEDGMENTS}

The authors would like to thank A. Weber, R. Schelldorfer, A. Young, M. Marcus, and A. Doran for their support. This work was supported by the Swiss National Science Foundation and measurements were performed at the Swiss Light Source at the Paul Scherrer Institute. In addition, the research leading to these results has received funding from the European Union's Seventh Framework Programme (FP7/2007-2011) under the Marie Skłodowska-Curie Grant Agreement No. 290605 (PSIFELLOW/COFOUND).
[1] L. Pauling, J. Amer. Chem. Soc. 57, 2680 (1935).

[2] M. J. Harris, S. T. Bramwell, D. F. McMorrow, T. Zeiske, and K. W. Godfrey, Phys. Rev. Lett. 79, 2554 (1997).

[3] A. P. Ramirez, A. Hayashi, R. J. Cava, R. Siddharthan, and B. S. Shastry, Nature (London) 399, 333 (1999).

[4] C. Castelnovo, R. Moessner, and S. L. Sondhi, Nature (London) 451, 42 (2008).

[5] J. S. Helton, K. Matan, M. P. Shores, E. A. Nytko, B. M. Bartlett, Y. Yoshida, Y. Takano, A. Suslov, Y. Qiu, J.-H. Chung, D. G. Nocera, and Y. S. Lee, Phys. Rev. Lett. 98, 107204 (2007).
[6] D. Pomaranski, L. R. Yaraskavitch, S. Meng, K. A. Ross, H. M. L. Noad, H. A. Dabkowska, B. D. Gaulin, and J. B. Kycia, Nat. Phys. 9, 353 (2013).

[7] A. S. Wills, R. Ballou, and C. Lacroix, Phys. Rev. B 66, 144407 (2002).

[8] Y. Tabata, H. Kadowaki, K. Matsuhira, Z. Hiroi, N. Aso, E. Ressouche, and B. Fåk, Phys. Rev. Lett. 97, 257205 (2006).

[9] Y. Qi, T. Brintlinger, and J. Cumings, Phys. Rev. B 77, 094418 (2008). 
[10] E. Mengotti, L. J. Heyderman, A. Fraile Rodríguez, F. Nolting, R. V. Hügli, and H. B. Braun, Nat. Phys. 7, 68 (2011).

[11] N. Rougemaille, F. Montaigne, B. Canals, A. Duluard, D. Lacour, M. Hehn, R. Belkhou, O. Fruchart, S. El Moussaoui, A. Bendounan, and F. Maccherozzi, Phys. Rev. Lett. 106, 057209 (2011).

[12] A. Farhan, P. M. Derlet, A. Kleibert, A. Balan, R. V. Chopdekar, M. Wyss, L. Anghinolfi, F. Nolting, and L. J. Heyderman, Nat. Phys. 9, 375 (2013).

[13] A. Farhan, A. Kleibert, P. M. Derlet, L. Anghinolfi, A. Balan, R. V. Chopdekar, M. Wyss, S. Gliga, F. Nolting, and L. J. Heyderman, Phys. Rev. B 89, 214405 (2014).

[14] I. A. Chioar, B. Canals, D. Lacour, M. Hehn, B. Santos Burgos, T. O. Menteş, A. Locatelli, F. Montaigne, and N. Rougemaille, Phys. Rev. B 90, 220407(R) (2014).

[15] S. Zhang, I. Gilbert, C. Nisoli, G. W. Chern, M. J. Erickson, L. O. Brien, C. Leighton, P. E. Lammert, V. H. Crespi, and P. Schiffer, Nature (London) 500, 553 (2013).

[16] L. J. Heyderman and R. L. Stamps, J. Phys.: Condens. Matter. 25, 363201 (2013).

[17] C. Nisoli, R. Moessner, and P. Schiffer, Rev. Mod. Phys. 85, 1473 (2013).

[18] G. Möller and R. Moessner, Phys. Rev. B 80, 140409(R) (2009).

[19] G. W. Chern, P. Mellado, and O. Tchernyshyov, Phys. Rev. Lett. 106, 207202 (2011).
[20] M. E. Brooks-Bartlett, S. T. Banks, L. D. C. Jaubert, A. HarmanClarke, and P. C. W. Holdsworth, Phys. Rev. X 4, 011007 (2014).

[21] B. Canals, I.-A. Chioar, V.-D. Nguyen, M. Hehn, D. Lacour, F. Montaigne, A. Locatelli, T. O. Mentes, B. S. Burgos, and N. Rougemaille, Nat. Commun. 7, 11446 (2016).

[22] L. Anghinolfi, H. Luetkens, J. Perron, M. G. Flokstra, O. Sendetskyi, A. Suter, T. Prokscha, P. M. Derlet, S. L. Lee, and L. J. Heyderman, Nat. Commun. 6, 8278 (2015).

[23] S. Ladak, D. E. Read, G. K. Perkins, L. F. Cohen, and W. R. Branford, Nat. Phys. 6, 359 (2010).

[24] A. Farhan, P. M. Derlet, A. Kleibert, A. Balan, R. V. Chopdekar, M. Wyss, J. Perron, A. Scholl, F. Nolting, and L. J. Heyderman, Phys. Rev. Lett. 111, 057204 (2013).

[25] V. Kapaklis, U. B. Arnalds, A. Farhan, R. V. Chopdekar, A Balan, A. Scholl, L. J. Heyderman, and B. Hjövarsson, Nat. Nanotechnol. 9, 514 (2014).

[26] L. Le Guyader, A. Kleibert, A. Fraile Rodríguez, S. El Moussaoui, A. Balan, M. Buzzi, J. Raabe, and F. Nolting, J. Elec. Spec. Rel. Phenom. 185, 371 (2012).

[27] A. Doran, M. Church, T. Miller, G. Morrison, A. T. Young, and A. Scholl, J. Elec. Spect. Rel. Phenom. 185, 340 (2012).

[28] J. Stöhr, Y. Wu, B. D. Hermsmeier, M. G. Samant, G. R. Harp, S. Koranda, D. Dunham, and B. P. Tonner, Science 259, 658 (1993).

[29] W. M. Young and E. W. Elcock, Proc. Phys. Soc. 89, 735 (1966). 